\title{
Glucocorticoid Resistance
}

National Cancer Institute

\section{Source}

National Cancer Institute. Glucocorticoid Resistance. NCI Thesaurus. Code C126809.

An autosomal dominant condition due to mutation(s) in the NR3C1 gene, encoding the glucocorticoid receptor, resulting in decreased receptor affinity to glucocorticoids.

Compensatory activation of the hypothalamic- pituitary-adrenal (HPA) axis results in increased mineralocorticoid and androgen production; clinical manifestations of glucocorticoid deficiency are rare. 